\title{
Using Molecular Dynamics Simulations to Model the DC Electrical Behavior of Confined Layers
}

\author{
Nathan G. Beougher, Jason Mclntosh, Jason A. Djuren, and M.W. Roth \\ Department of Physics \\ University of Northern lowa \\ Cedar Falls, lowa 50614 USA
}

\author{
Carlos Wexler \\ Department of Physics and Astronomy \\ University of Missouri \\ Columbia, Missouri 65211 USA
}

\author{
Carl Thurman \\ Department of Biology \\ University of Northern lowa \\ Cedar Falls, lowa 50614-0421 USA
}

Received: May 8, $2006 \quad$ Accepted: May 20, 2006

\begin{abstract}
A Molecular Dynamics (MD) computer simulation is utilized to qualitatively understand the DC electrical conduction behavior of pentadecane $\left(\mathrm{C}_{15} \mathrm{H}_{32}\right)$ layers confined between two graphite slabs as related to the dynamics of the layer. At low temperatures the patch remains together and perpendicular to the confining layers. Then, as temperature is increased, tilting of the molecules begins. The molecules tend to remain straight as they tilt with increasing temperature which, in our model, affects the capacitance somewhat but the resistance little. As temperature is increased further, the molecules exhibit gauche defects which accompany patch collapse. During patch collapse, the system shows dramatic changes in its calculated DC resistance and capacitance. Calculated specific capacitance values are in remarkable agreement with recent experimental measurements. Results for two different confining layer separations are discussed as well as future work related to lipid bilayer systems.
\end{abstract}

\section{INTRODUCTION}

Biological membranes are composed of a combination of proteins, carbohydrates, and lipids. These lipids form stable bilayer structures and are present in animal and plant matter. The structure of lipid bilayer membranes is typically comprised of varied head groups and hydrocarbon tails [1]. Of interest in studies of electrical properties of lipid bilayers are membranes which have highly polar head groups, due in part to the geometry of such bilayer systems solvated in water. The structure of lipid bilayer membranes lends itself to exhibiting two key DC electrical properties: capacitance and resistance. When constructed as a bilayer system, the polar head groups may be considered to be attached at opposite ends with the hydrocarbon chains stretching towards each other in the space between the opposing polar head attachments. Considering the distance between the placement of one layer of molecules and the respect to the placement of the other layer, a gap will likely exist between the hydrocarbon chains. The chains can be considered as tails of the molecules that contribute to the resistance of the system. In addition, the gap between the molecules must also contribute to the 
overall resistance, as electrons must tunnel through the space.

A currently active area of scientific research involves characterizing the DC electrical behavior of lipid bilayer membranes. Although hydrodynamic and other material properties of biological membranes have been studied, little is known about their electrical behavior. One way to probe the electrical behavior of biological membranes is to study the effect of adding other molecules. Recent experimental measurements [2] of the effect of the concentration of cholesterol added to an artificial 1,2-dioleoyl-sn-glycero-3phosphocholine (DOPC) at $T=296 \mathrm{~K}$ gave the surprising result that cholesterol concentration did not affect the conductance. The system's resistance stayed at about $35.1 \mathrm{G} \Omega$ and its specific capacitance at ca. $0.38 \mu \mathrm{f} / \mathrm{cm}^{2}$.[2]

In order to better understand the dymanics of biological membranes in relation to its $D C$ electrical properties, we feel that it is crucial to thoroughly characterize simpler systems which represent only the hydrophobic tails first. Alkanes are chosen, because they are a family of hydrophobic chain - like molecules that differ mainly in their length. Moreover, confined alkane systems are interesting in their own right [3-4], and calculations [5] show that perpendicular tetracosane layers on a single sheet of graphite undergo a patch collapse, which would exhibit strong signatures in the system's capacitance and resistance. In particular, we use patches of pentadecane $\left(\mathrm{C}_{15} \mathrm{H}_{32}\right.$, or $\left.\mathrm{C} 15\right)$ sandwiched between two graphite slabs. Such choices are motivated by the fact that $\mathrm{C} 15$ shows a strong perpendicular phase that persists through a wide temperature range, and graphite is chemically inert, mechanically stable and widely available as a substrate. The purpose of this work is to use the results of Molecular Dynamics (MD) computer simulations of the confined $\mathrm{C}_{15} \mathrm{H}_{32}$ layers to relate the system dynamics to the DC electrical properties.

The dynamics that result from our MD simulations can be used in a macroscopic effective circuit model to characterize and better understand the temperature dependence of the system's resistance and capacitance. The general geometry of the system is that of series resistors and capacitors, as shown in Figure 1. Here we assign the widely used dielectric constant $\mathrm{k}=3$ to the alkane layer, which for
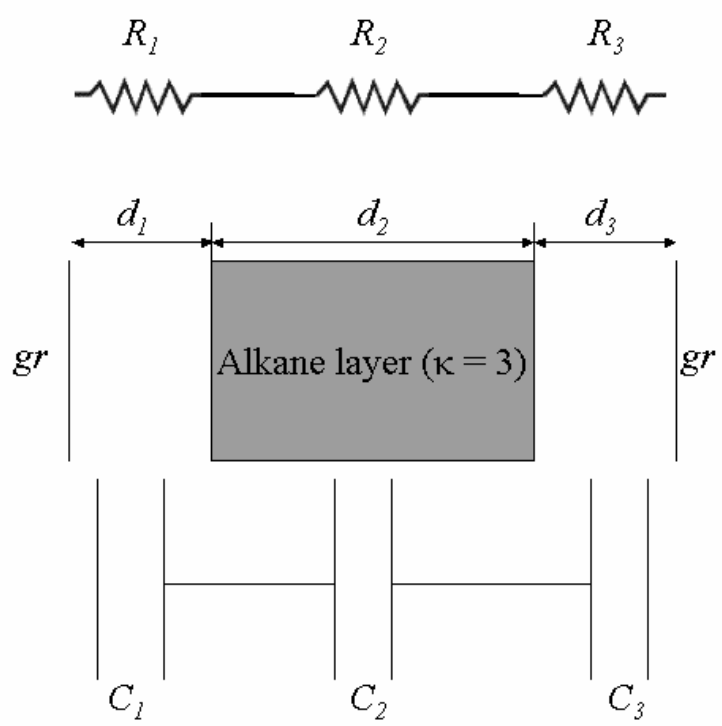

Figure 1. Reduced circuit representing the confined alkane system. The dark rectangle represents the alkane film which is assumed to occupy the entire area of the slabs, and effective resistive and capacitive schematics are shown above and below, respectively. 


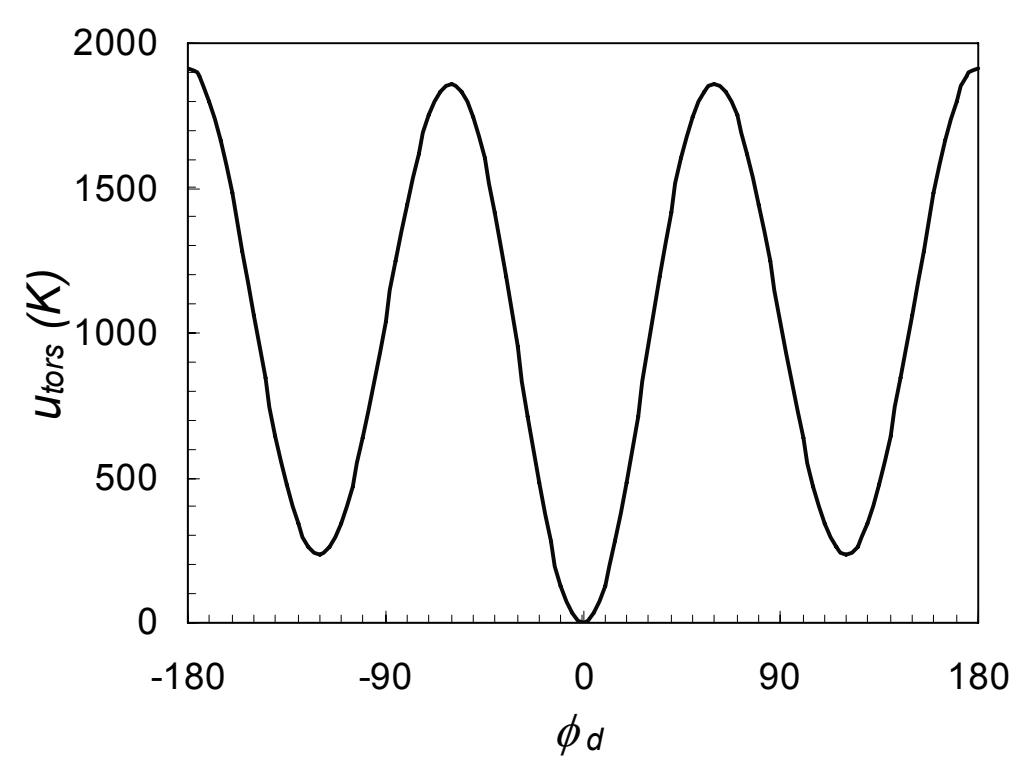

Figure 2. Torsional potential $u_{\text {tors }}(\varphi)$. The absolute minimum at $\varphi=0^{\circ}$ corresponds to the trans configuration, where three sequential bonds are co-planar and the hydrogens of the methyl or methylene groups are as far apart as possible. The two local minima at $\varphi= \pm 120^{\circ}, u_{\text {tors }}=234 \mathrm{~K}$, are the gauche configurations. If one or more gauche defects are present, the molecule is not planar.

ease of calculation we assume to occupy the entire area between the graphite slabs. Our model then suggests that the resistance for the system is of the following form

$$
R=A e^{\alpha d_{1}}+B e^{\beta d_{2}}+C e^{\alpha d_{3}}
$$

where the exponential increase of each term with distance applies to tunneling current as well as current through the molecules. Also, $(A, B, C, \alpha, \beta)$ are constant parameters and $\left(d_{1}, d_{2}, d_{3}\right)$ are distances obtained from thermal averages of the dynamical computer simulations. Since we have no accurate estimates of the constant parameters, we chose $B$ to be much less than $A=C$, and also $\alpha$ about twice as large as $\beta$, which is to say that the regions of empty space contribute much more to the resistance than do the molecules themselves. The capacitors are treated as parallel plate capacitors in series. Since the surface area is not uniquely determined, we are only interested in the specific capacitance:

$$
\frac{C}{A}=\frac{\varepsilon_{o}}{d_{1}+\frac{d_{2}}{\kappa}+d_{3}} \text {. }
$$

\section{ABOUT THE SIMULATIONS}

The first of the non-bonded interactions is the adsorbate-adsorbate interaction, and is modeled by the well known Lennard Jones pair potential function

$$
u_{L J}\left(r_{i j}\right)=4 \varepsilon_{i j}\left[\left(\frac{\sigma_{i j}}{r_{i j}}\right)^{12}-\left(\frac{\sigma_{i j}}{r_{i j}}\right)^{6}\right] .
$$

In eqn. (3), $\varepsilon$ refers to the well depth of the potential, and $\sigma$ represents the collision diameter. Lorentz-Bertholot combining rules

$$
\sigma_{i j}=\frac{\sigma_{i}+\sigma_{j}}{2}, \quad \varepsilon_{i j}=\sqrt{\varepsilon_{i} \varepsilon_{j}},
$$

are used to describe mixed interactions when particles $i$ and $j$ are of different types.

The other non-bonded potential used is the graphite surface potential, given by a Fourier expansion proposed by Steele [6]

$$
u_{i}^{g r}=E_{0 i}\left(z_{i}\right)+\sum_{n=1}^{\infty} E_{n i}\left(z_{i}\right) f_{n i}\left(x_{i}, y_{i}\right),
$$

where 


$$
\begin{gathered}
E_{0 i}\left(z_{i}\right)=\frac{2 \pi q \varepsilon_{g r} \sigma_{g r}^{6}}{a_{s}}\left(\frac{2 \sigma_{g r}^{6}}{45 d\left(z_{i}+0.72 d\right)^{9}}+\frac{2 \sigma_{g r}^{6}}{5 z_{i}^{10}}-\frac{1}{z_{i}^{4}}-\frac{2 z_{i}^{2}+7_{z_{i}} d+7 d^{2}}{6 d\left(z_{i}+d\right)^{5}}\right), \\
E_{n i}\left(z_{i}\right)=\frac{2 \pi \varepsilon_{g r} \sigma_{g r}^{6}}{a_{s}}\left[\left(\frac{\sigma_{g r}^{6}}{30}\right)\left(\frac{g_{n}}{2_{z_{i}}}\right)^{5} K_{5}\left(g_{n} z_{i}\right)-2\left(\frac{g_{n}}{2_{z_{i}}}\right)^{2} K_{2}\left(g_{n} z_{i}\right)\right],
\end{gathered}
$$

and

$$
f_{1}\left(x_{i}, y_{i}\right)=-2 \cos \left[\frac{2 \pi}{a}\left(x+\frac{y}{\sqrt{3}}\right)\right]-2 \cos \left[\frac{2 \pi}{a}\left(x-\frac{y}{\sqrt{3}}\right)\right]-2 \cos \left[\frac{4 \pi}{a}\left(\frac{y}{\sqrt{3}}\right)\right] \text {. }
$$

Here $g_{n}$ is the modulus of the $n^{\text {th }}$ graphite reciprocal lattice vector and the $K$ 's are modified Bessel functions of the second kind. The interaction is obtained by summing over an infinite number of graphene sheets using the Euler-MacLaurin Theorem. Only $f_{1}\left(x_{i}, y_{i}\right)$ is defined above because the sum in equation (4) converges rapidly and only the $n$ $=1$ term is used. All important potential parameters are identified in Table I.

To include the second graphite slab placed at a height $\mathrm{h}$ the total interaction of atom $(i)$ with the graphite is then expressed as

$$
u_{i}^{g r}\left(\vec{r}_{i}\right)=u_{i}^{g r, b o t}+u_{i}^{g r, t o p}=E_{0 i}\left(z_{i}\right)+E_{0 i}\left(h-z_{i}\right)+\left\{E_{1 i}\left(z_{i}\right)+E_{1 i}\left(h-z_{i}\right)\right\} f_{1}\left(x_{i}, y_{i}\right) .
$$

In this work, there are two bonded interactions that are used, bond angle bending and dihedral angle bending (torsion). All bond lengths are held constant at $1.54 \AA$ with the RATTLE algorithm [7], which allows for constrained solution of the equations of motion. The first bonded interaction is bond angle bending. Assuming the bond angles to be harmonic, the potential can be expressed as [8]

$$
u_{\text {bend }}=\frac{1}{2} k_{\theta}\left(\theta_{b}-\theta_{0}\right)^{2} \text {, }
$$

where $\theta_{b}$ is the bond angle, $\theta_{0}$ is the equilibrium bond angle and $k$ is the angular stiffness. The other bonded interaction is dihedral (torsional) bending, which is of the form [9]

$$
u_{\text {tors }}=\sum_{i=0}^{5} c_{i}\left(\cos \phi_{d}\right)^{i}
$$

where $\phi_{c l}$ is the dihedral angle and the $c_{i}$ are constants. Figure 2 shows the torsional potential and describes the types of configurations found. Parameters for bonded potentials are in Table 2 .

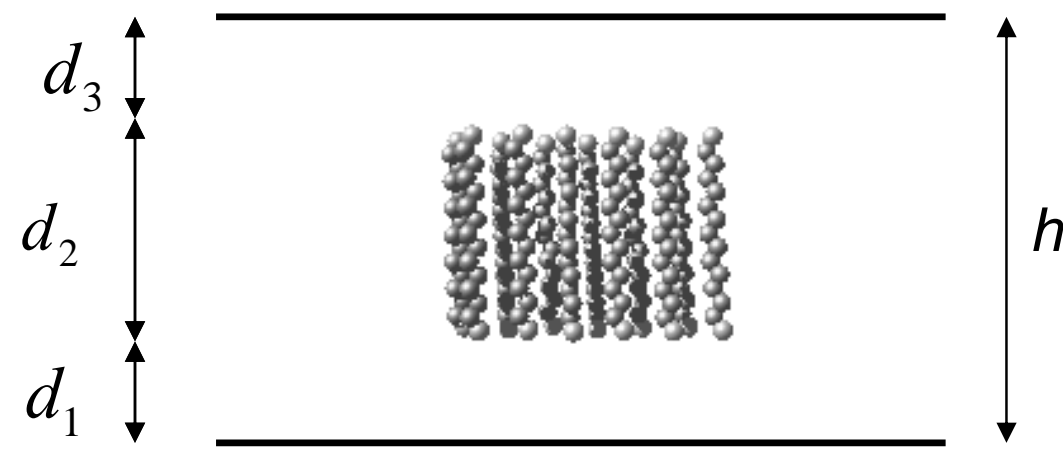

Figure 3. Initial configuration of the confined alkane system. Less than 90 molecules are shown here to aid in visualization. 


\begin{tabular}{|c|c|}
\hline Parameter & Value \\
\hline$\varepsilon_{\mathrm{CH} 3-\mathrm{CH} 3}$ & $72 \mathrm{~K}$ \\
\hline$\sigma_{\mathrm{CH} 3-\mathrm{CH} 3}$ & $3.923 \AA$ \\
\hline$\varepsilon_{\mathrm{CH} 2-\mathrm{CH} 2}$ & $72 \mathrm{~K}$ \\
\hline$\sigma_{\mathrm{CH} 2-\mathrm{CH} 2}$ & $3.923 \AA$ \\
\hline$Q$ & 2 \\
\hline$a_{s}$ & $5.24 \AA^{2}$ \\
\hline$D$ & $3.357 \AA$ \\
\hline$\varepsilon_{\mathrm{gr}}$ & $44.89 \mathrm{~K}$ \\
\hline$\sigma_{\mathrm{gr}}$ & $3.66 \AA$ \\
\hline
\end{tabular}

Table 1. Non-bonded potential parameters used in the simulations.

The system initially has $90 \mathrm{C}_{15} \mathrm{H}_{32}$ molecules placed perpendicularly between two graphite slabs as shown in Figure 3. Two values of graphite slab separation are used: $h=30.7 \AA h=32.7 \AA$. After the initial conditions are realized the system is advanced through time utilizing a standard Verlet integration scheme with a time step $\Delta t$ $=1 \mathrm{ps}$. Each system is equilibrated for $2 \times 10^{4}$ steps and important averages are subsequently taken over the next $6 \times 10^{4}$ time steps.

\section{Results and Discussion}

Figure 4 shows the average end- to -end molecule length and average patch thickness as functions of temperature for $h=$ $30.7 \AA$. Based upon the data shown as well as that for other values of $h$, there seems to be three distinct regimes with different behavior. The first corresponds to the low temperature range ( $T=20 \mathrm{~K}$ to $275 \mathrm{~K}$ ) when the curves in the plot are almost flat. In this regime, the molecules start out in a perpendicular phase and then tilt. The observation that the average end-to-end length remains flat but the patch thickness dips a little confirms that the molecules are tilting but remaining straight. The calculated resistance and specific capacitance of the system in Figure 5 show that slight patch thickness change in the low temperature affects the resistance little but the capacitance to a greater extent.

The next significant change the system undergoes is from $T=275 \mathrm{~K}$ to $325 \mathrm{~K}$ where the patch collapses. Here the plot in Figure 4 shows that as the thickness of the patch dramatically decreases the molecular end-to-end separation also decreases, signifying the presence of gauche defects, where the molecules twist about a bond. The increased empty space causes the resistance to sharply increase; the capacitance decreases because less space is being occupied by the dielectric medium. Subject to appropriate fine tuning, and considering that the resistance is plotted on a logarithmic scale it seems imaginable that the patch collapse could be used to turn a circuit off and on.

As illustrated in Figure 4, the final temperature regime $(T>325 \mathrm{~K})$ involves the collapsed physisorbed patch undergoing increasing thermal fluctuations as temperature rises. It is interesting to note that the capacitance increases and the resistance decreases in this regime. Such behavior can be explained in the following way: excursions of the molecules bound to the substrate increase in magnitude with increasing temperature, which in a time average has the effect of thickening the patch. Therefore the patch faces are both closer to the graphite planes and the patch occupies more space. The former consideration explains a drop in resistance through the tunneling contribution and the latter explains an increase in capacitance because the molecules have a dielectric constant three times that of empty space.

The simulation results are very sensitive to graphite sheet spacing, as for most separations the molecules will tilt even in the low temperature regime. Figure 6 (page 8) shows the average end-to-end molecule length and average patch thickness as functions of temperature for a

\begin{tabular}{|c|c|}
\hline Parameter & Value \\
\hline$k_{\theta}$ & $62793.59 \mathrm{~K} / \mathrm{rad}^{2}$ \\
\hline$\theta_{0}$ & $114^{\circ}$ \\
\hline$c_{0}$ & $1037.76 \mathrm{~K}$ \\
\hline$c_{1}$ & $2426.07 \mathrm{~K}$ \\
\hline$c_{2}$ & $81.64 \mathrm{~K}$ \\
\hline$c_{3}$ & $-3129.46 \mathrm{~K}$ \\
\hline$c_{4}$ & $-163.28 \mathrm{~K}$ \\
\hline$c_{5}$ & $-252.73 \mathrm{~K}$ \\
\hline
\end{tabular}

Table 2. Bonded potential parameters used in the simulation. 


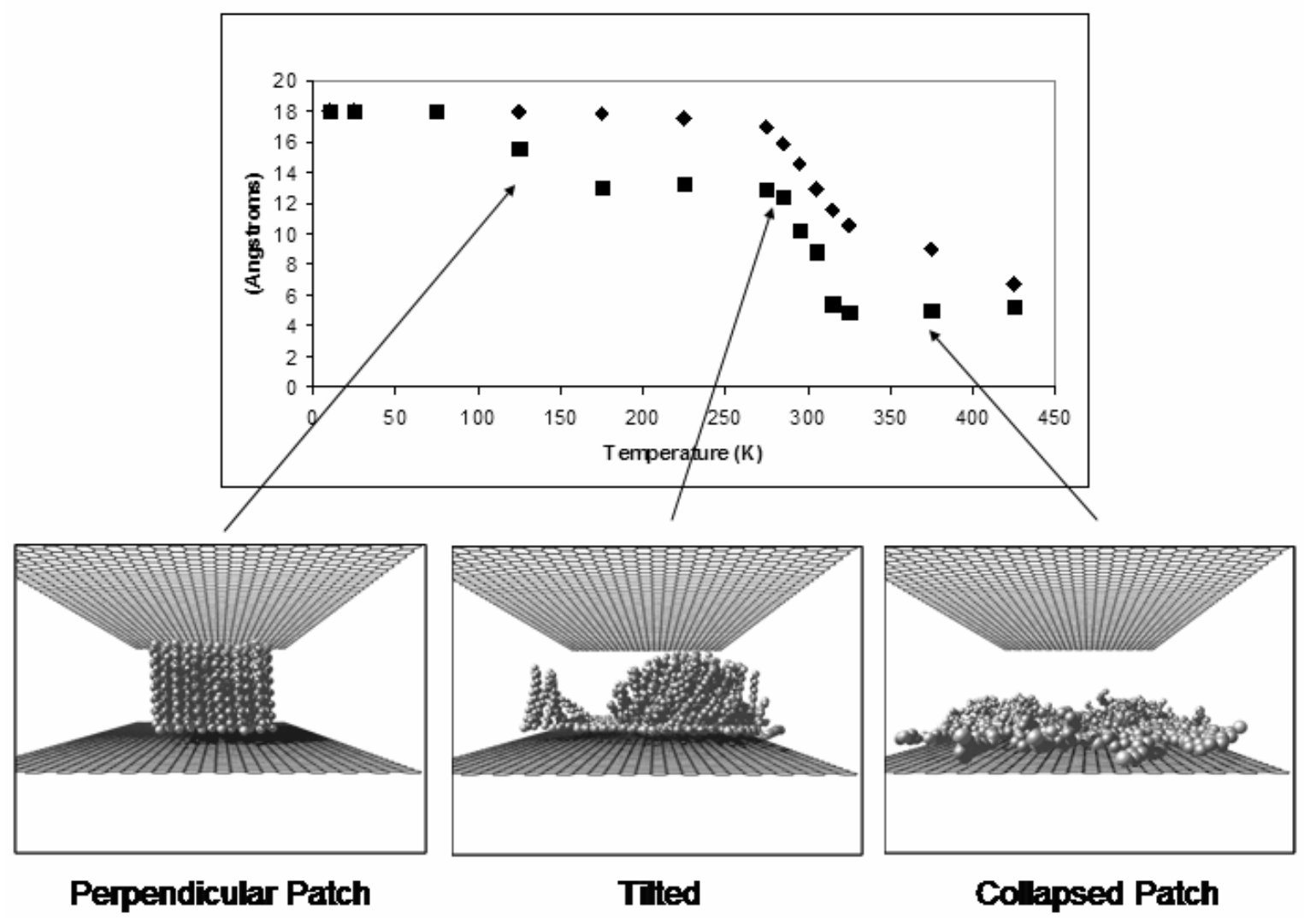

Figure 4. Average end-to-end molecule lengths (upper graph; diamonds), average patch thickness (upper graph; squares) and ray traced renderings of final configurations corresponding to three distinct temperature regions for the system's behavior at $h=30.7 \AA$.

graphite plane separation $h=32.7 \AA$. It is apparent that the three regions of unique system behavior discussed above are still present, but the patch collapse is much more gradual than for $h=32.7$ and is extended on the high temperature side of the transition. Such behavior can be partially understood through realizing that, because tilting is more prevalent early on in temperature, the patch tends to collapse more onto itself here rather than collapsing directly on the graphite substrate.

The calculated specific capacitance from this study is in remarkable agreement with the measured value for lipid bilayer systems[2] of ca. $0.38 \mu \mathrm{F} / \mathrm{cm}^{2}$. This suggests that we may be able to understand some processes (related to gaps between molecules) in more complicated systems using models of simpler ones. For all temperature ranges we wish to emphasize that our estimates for the system's capacitance are much more reliable than those for the resistance because a priori we don't know exactly how much more the tunneling contributes than the molecular resistance. If the relative strengths of the resistance contributions are very different than the values used in this work, then the details of the curve could be much different from any real system although the fundamental trends still are expected to be reasonable. Also, it seems difficult to compare the results of this work to those of recent experimental efforts [2] mainly because the latter involves a study where temperature is held fixed at $T=296 \mathrm{~K}$. With more experimental data and improved MD simulations it is expected that our understanding of biological membranes on the nanoscale will dramatically improve.

Obviously real biological systems (lipid bilayers) have molecules with much more complicated architecture than presented in this study and will require larger simulation sizes and longer 

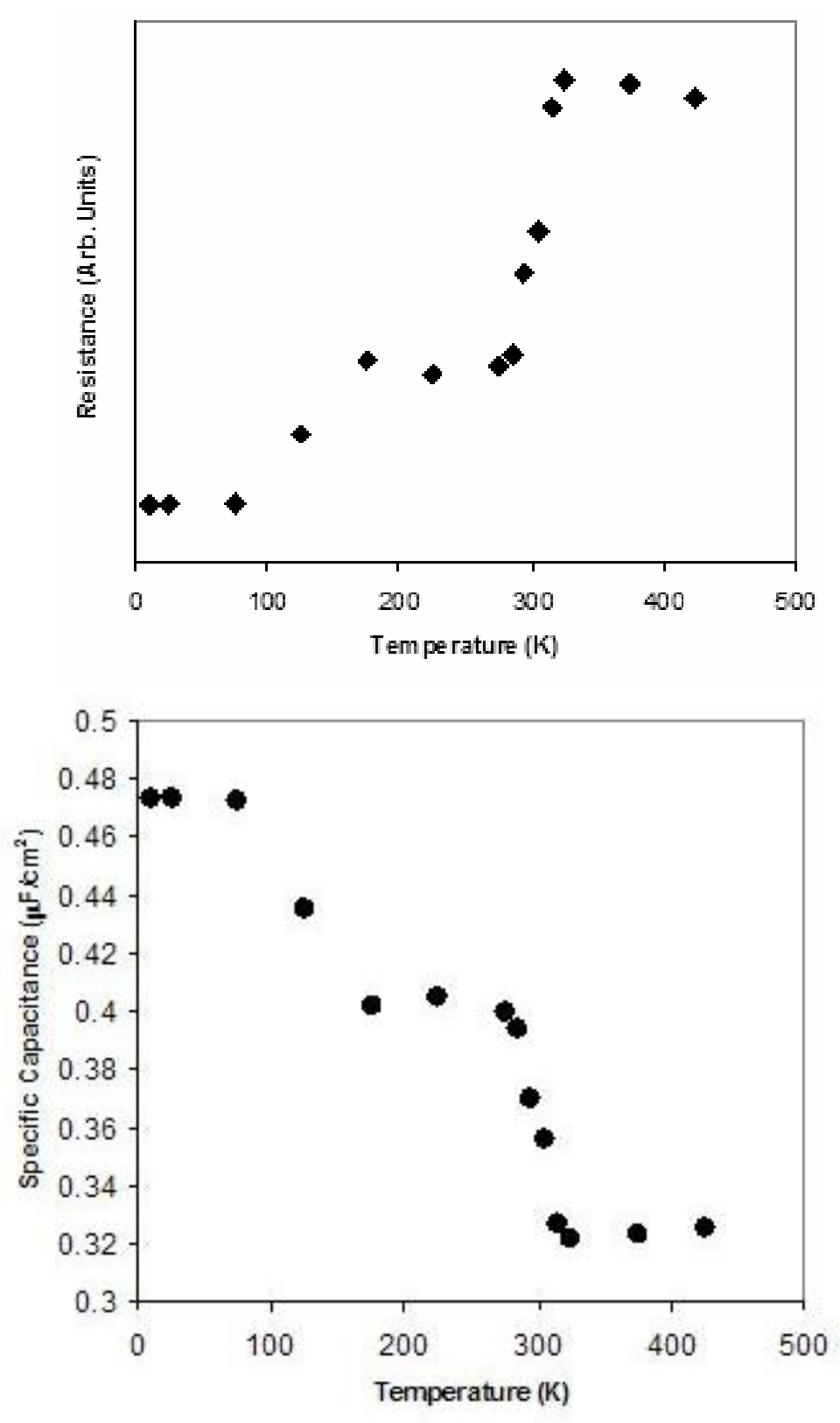

Figure 5. Calculated resistance (top) and specific capacitance (bottom) as functions of temperature for $h=30.7 \AA$.

computational times. An obvious extension of our work would be to study two patches placed end - to - end between graphite slabs. Since the methyl groups adsorb strongly to the graphite, focusing on the dynamics present at the gap between the patches would be most interesting. Subsequently, simulation of a lipid bilayer membrane solvated in water is planned, again focusing on the dynamics present at the gap between the hydrophobic tails.

\section{ACKNOWLEDGEMENTS}

The authors wish to thank the University of Northern lowa Physics Department for supporting this research with an undergraduate summer 2003 fellowship, as 


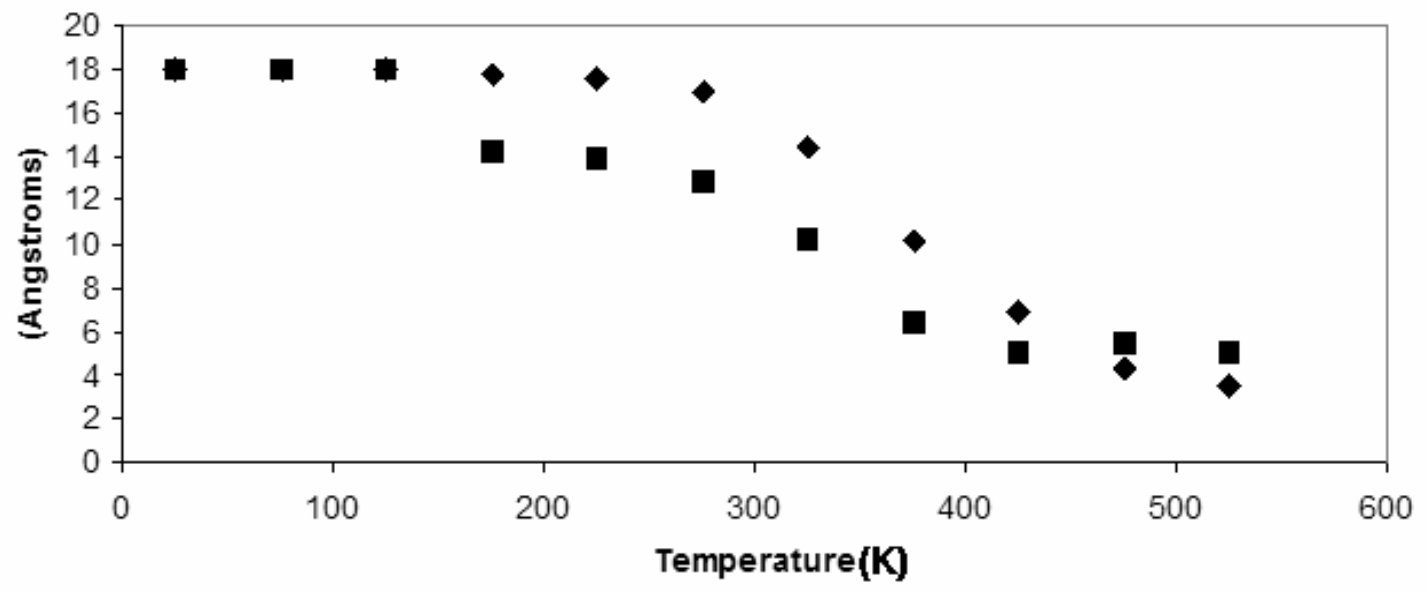

Figure 7. Average end-to-end molecule lengths (diamonds), average patch thickness (squares) $h=30.7 \AA$.

well as the Biology and Chemistry Departments for sharing their Merck/AAAS support. Also, the authors acknowledge rich and fruitful discussions with Flemming Hansen and Hak Taub.

\section{REFERENCES}

1. A.L. Rabinovich, P.O. Ripatti, N.K. Balabaev, and F.A.M. Leermakers. Physical Review E. 67, 011909 (2003).

2. Jason McIntosh, Carl Thurman, John Schoer and Michael Roth, "Nanoelectronics of Planar Lipid Bilayer Membranes" (Unpublished Technical Report)

3. Hugo K. Christensesn, J. Phys: Cond. Matt., 13, R95 (2001)
4. C.L. Pint and Michael W. Roth, Proceedings of the Fall 2005 Materials Research Society Meeting, paper \#0899-N07-21.

5. Michael Roth and Carlos Wexler, "Molecular Dynamics Simulations of Perpendicular Tetracosane Films", 2006 APS March Meeting, Baltimore, MD (3/15/06).

6. W.A. Steele, Surf. Sci. 36, 317 (1973).

7. M. P. Allen, D. J. Tildesley, (Clarendon Press, New York, 1988).

8. M. G. Martin and J. I. Siepmann, J. Phys. Chem. 102, 2569 (1998).

9. P.Padilla and S. Toxværd, J. Chem. Phys. 945650 (1991).

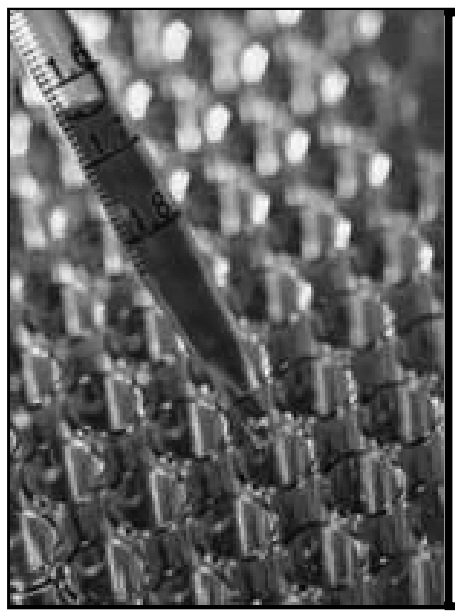

\section{Merck $\downarrow$ AAAS Undergraduate Science Research Program}

The Merck/AAAS Undergraduate Science Research Program (USRP) is a national competitive awards program available in all 50 states, the District of Columbia, and Puerto Rico. Up to 15 new awards will be made annually. Each award provides up to $\$ 60,000$ paid over three years at $\$ 20,000$ per year for joint use by the biology and chemistry departments at each recipient institution. The funding supports research stipends for undergraduate students and ancillary programs that foster interactions between these departments.

http://www.merckaaasusrp.org/ 


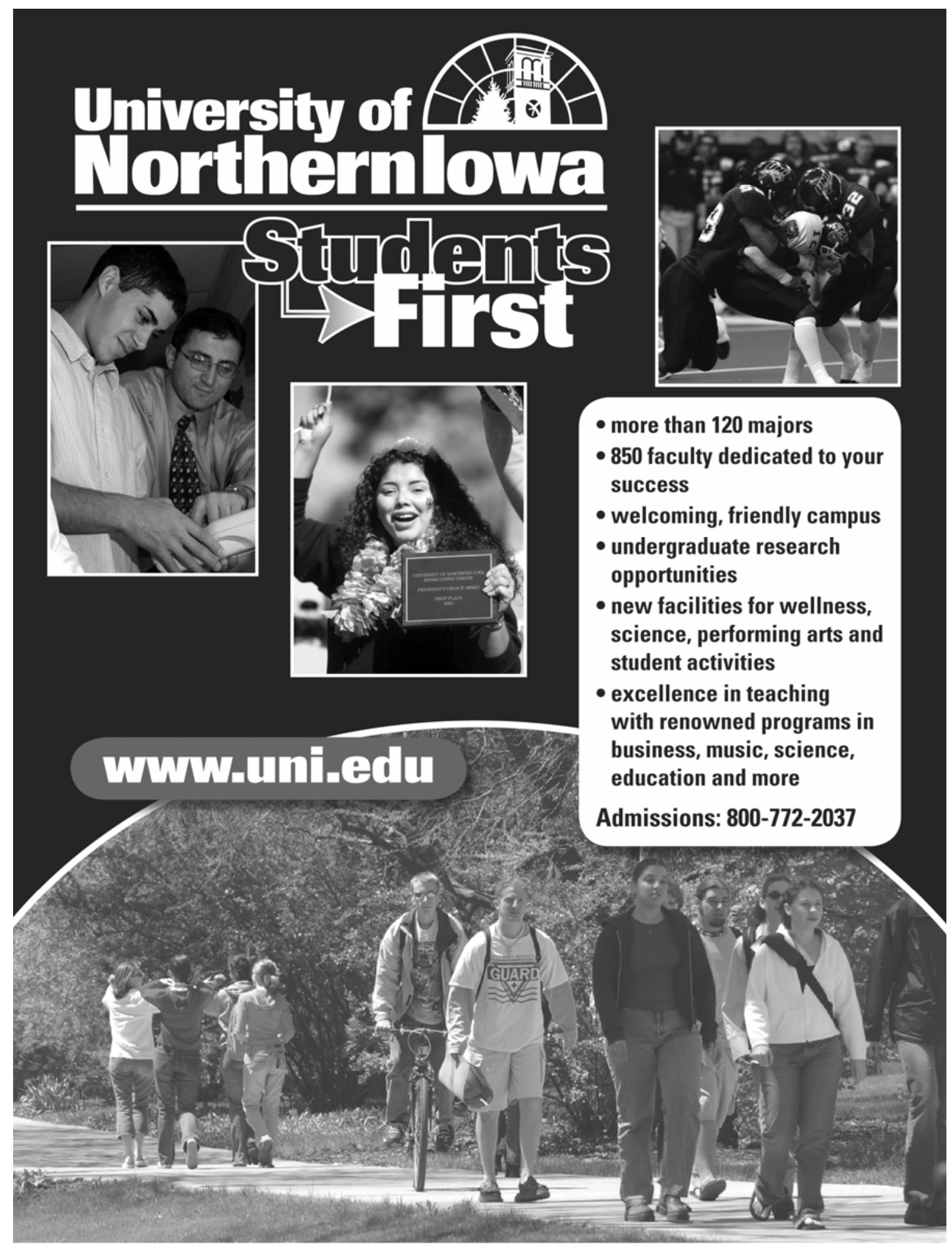




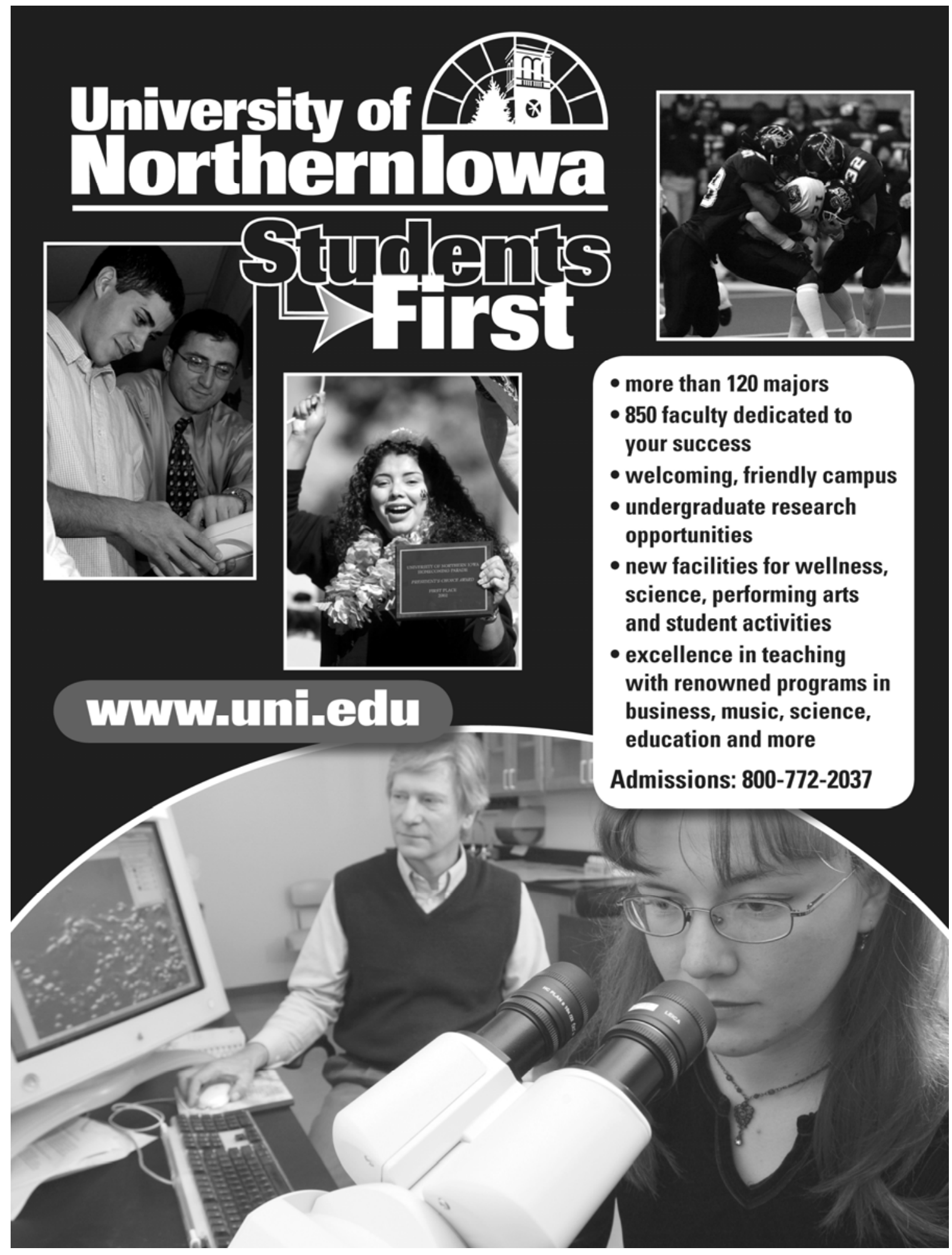

